University of South Carolina

Scholar Commons

7-1996

\title{
The Effects of a 3-Week Unit of Tactical, Skill, or Combined Tactical and Skill Instruction on Badminton Performance of Ninth- Grade Students
}

\author{
Karen E. French \\ University of South Carolina - Columbia, kfrench@mailbox.sc.edu \\ Peter H. Werner \\ Judith E. Rink \\ University of South Carolina -Columbia, jrink@mailbox.sc.edu \\ Kevin Taylor \\ University of Northern Colorado \\ Kevin Hussey \\ Eastern Illinois University
}

Follow this and additional works at: https://scholarcommons.sc.edu/pedu_facpub

Part of the Education Commons

\begin{abstract}
Publication Info
Published in Journal of Teaching in Physical Education, Volume 15, Issue 4, 1996, pages 418-438. http://journals.humankinetics.com/jtpe-contents (C) 1996 by Human Kinetics Publishers
\end{abstract}

This Article is brought to you by the Physical Education, Department of at Scholar Commons. It has been accepted for inclusion in Faculty Publications by an authorized administrator of Scholar Commons. For more information, please contact digres@mailbox.sc.edu. 


\title{
The Effects of a 3-Week Unit of Tactical, Skill, or Combined Tactical and Skill Instruction on Badminton Performance of Ninth-Grade Students
}

\author{
Karen E. French, Peter H. Werner, Judith E. Rink \\ University of South Carolina
}

\author{
Kevin Taylor \\ University of Northern Colorado
}

\author{
Kevin Hussey \\ Eastern Illinois University
}

Interest in alternative models for games/sport instruction has increased in recent years. Recent research has compared tactical approaches to games/sport instruction with more skill-oriented approaches (Gabriele \& Maxwell, 1995; Griffin, Olsin, \& Mitchell, 1995; McPherson \& French, 1991; Turner \& Martinek, 1992, 1995b). Most of the research in this area to date has focused on student performance outcomes (knowledge, skill, game play performance) that result from different skill and tactical approaches to instruction and learning.

Rink, French, and Tjeerdsma (1996) describe several design problems inherent in conducting and reporting research on skill and tactical awareness approaches to teaching games/sport. The first issue is related to the inclusion of game play in the treatment. Game play was included as a form of instruction for the skill group in McPherson and French (1991) and Turner and Martinek (1995b). In other studies (Gabriele \& Maxwell, 1995; Griffin, Olsin, \& Mitchell, 1995), the issue is unclear. The decision was made to include game play as a form of instruction for all treatment groups in the present badminton study.

The second design issue was related to the timing of the introduction of explicit skill instruction. Bunker and Thorpe (1982) advocate introducing explicit skill technique instruction when students demonstrate the need for skill or when poor skill technique limits tactics. Turner and Martinek (1995b) used a true game for understanding approach, and skill technique was introduced when students demonstrated the need for skill instruction. The introduction of skill and tactical instruction was controlled in McPherson and French (1991) and part of the research design.

The decision was made to control the amount of explicit instruction devoted to skill and tactics in the present study. The skill group received no explicit tactical instruction. Tactics were presented in the tactical group consistent with a games for understanding approach with the exception that no explicit skill instruction was introduced during tactical instruction. The combination group received explicit instruction in both skill and tactics. The combination group practiced tasks common to both the skill and tactical groups. The order of presentation of tactics and skill in the combination group did not follow a true games for understanding approach. For example, skill tasks were not presented in the combination group only 
when the need for the skill was limiting tactics. Thus, no treatment group in the present study followed a true games for understanding model.

Students may learn some aspects of skill and tactics without explicit instruction, whereas some aspects of performance may only improve with explicit direct instruction (McPherson \& French, 1991). A design that controlled the amount of explicit skill and tactical instruction would increase our understanding of which student outcomes may result from different forms of explicit instruction (skill or tactics), as well as outcomes that may be produced without explicit instruction.

\section{Methods}

\section{Participants}

Students. Forty-eight ninth-grade students were randomly selected from three high school physical education classes of approximately 90 students. Students were from a wide range of socioeconomic backgrounds, and approximately $40 \%$ were African American. Students had little previous experience in badminton. Twelve students were randomly assigned to each of three treatment groups and a control group. As a result of random assignment, the number of males and females in each group was similar.

Physical education was a required subject for students. Physical education classes for these students met for 55 minutes, 5 days per week. Instruction was typically conducted in 3-week units for a given activity.

Teachers. The teacher of the control group was a member of the high school physical education faculty. The teachers for each experimental group were the first three authors of the study (French, Werner, and Rink). Peter Werner taught the tactical group. He had previously studied with Almond, Bunker, and Thorpe while on sabbatical leave in Loughborough, England. He has written extensively on a games for understanding approach to teaching games, including a paper on teaching badminton using a games for understanding approach (Lawton \& Werner, 1989). In addition, he regularly teaches a course in education games (games for understanding approach, tactical principles which transfer across types of sports) to undergraduate physical education majors.

Judith Rink taught the skill group. She has written extensively on direct instruction of motor skills, and in her book (Rink, 1993), she outlines principles for direct instruction, content development, and game stages. Karen French, a former experienced public school teacher and now a university professor familiar with the issues related to the treatments, taught the combination group. Each of the experimental group teachers had considerable teaching experience in public schools prior to assuming positions in teacher education at the university level.

\section{Instruction and Treatment Verification}

The three treatment groups received instruction in badminton. Instruction in the skill group focused on badminton skills (i.e., serve, clear, drop, and smash). No explicit instruction of tactics was provided by the skill teacher. Instruction in the strategy group focused on tactical instruction. No explicit instruction of skill technique was provided by the tactical teacher. The combination group received 
explicit instruction in both badminton skills and tactics. The control group participated in a softball unit.

The unit of instruction was a 3-week, 15-lesson format. Due to unforeseen school assembly programs, 2 days of instruction were lost, so the unit was reduced to 13 days of instruction. On the first day of instruction, the unit was introduced to all experimental groups. A game played by two experienced badminton players was presented. Each teacher met with his or her respective group to learn names. A short period of play was conducted in which students hit a shuttle back and forth cooperatively with a partner. No formal instruction related to the treatments occurred the first day.

Following the introduction, each experimental group received instruction in badminton singles specific to their treatment for the remaining 12 lessons. All instruction concerned the game of singles. The experimental teachers met prior to the beginning of instruction to discuss their respective treatments. Teachers agreed to allocate the first 25 minutes of each lesson to instruction specific to their treatment. The remaining 15 minutes of each lesson were used for game play. Game play is a form of instruction. During game play for each lesson, teachers were limited to interventions during play that were consistent with their respective treatments. For example, the skill teacher could only intervene to give feedback or instruction on skill technique, the tactical teacher could only provide tactical feedback, and the combination teacher could provide feedback on skill technique or tactics during game play. Five minutes at the beginning and end of class was devoted to dressing and taking roll.

Instruction in the skill group focused on developing specific shots. On given days, the clear, drop, long and short serve, and smash were taught using direct instruction. The teacher refined the mechanics of each stroke concerning grip, arm and wrist action, body positioning and rotation, and footwork. Each skill was introduced and followed by extension and refinement task for each skill. The teacher intervened to provide feedback, a refining task, or both to direct attention to the technical aspects of the skill. Practice tasks became progressively more difficult. The teacher used several environmentally designed tasks. For example, students hit clear shots over ropes, beyond ropes placed on the floor near the center or back of the court, into hoops as targets. For the drop shot, students hit under a rope, sometimes into target areas in the corners of the court. Environmental extension tasks were used to focus student practice on aspects of the skill (e.g., trajectory, force production) and to elicit more mature movement patterns. Application tasks (count number of successful responses) were also used in conjunction with the environmental tasks. During game play, the skill teacher only provided feedback or instruction on skill technique.

The tactical group focused primarily on tactical concepts unique to net/wall games. Many of the tasks presented during instruction are described in more detail in Lawton and Werner (1992). Many of the tasks could be characterized as a more indirect approach to teaching tactics. Lawton and Werner (1989) also present suggestions where skill instruction should be added to modified games. In this study, no skill instruction was presented during instruction in the tactical group. Most tasks were presented in conjunction with a modified game form. Modified game forms were used to present tactics related to the need for service rule, how to score or win a point, hitting to open spaces, long and short game, left and right game, 
gaining an offensive advantage, analyzing an opponent's weakness, bisecting angles for defense, and buying time to recover from a weak shot. For example, modified games were played to reinforce and practice the long and short tactics. Three example games were played in which students scored an extra point each time (a) the shuttle hit the floor behind the opponent, (b) the shuttle was hit over the head of the opponent, or (c) the shuttle landed on the court between the net and service line. Other specific game forms used in practice focused on hitting the shuttlecock left then right, returning to home base after each shot, keeping the racket high and making contact as early as possible to hit down on the shuttlecock, and anticipating the opponent's position to outmaneuver him or her. During game play, the teacher only provided feedback on the tactical aspects of game play. On the last day of instruction, one task asked students to play an opponent and between points write on a piece of paper their plans for the next point, analysis of opponents strengths and weaknesses, and other tactics used during game play.

The combination group received instruction in both skills and tactics. Tasks selected for student practice included tasks practiced by the skills group (clear, drop, serve, and smash) and the tactical group (e.g., hitting down on the shuttle, hitting away from an opponent, long/short game, move opponent left and right). The skill tasks were presented using direct instruction. Each skill was introduced and followed by extension and refinement tasks for the skill. Some of the environmentally designed extension tasks used by the skill group were practiced (e.g., hit clear over rope, hit clear beyond rope in back of court, hit drop under rope or into hoop target areas in front corners). Tactical tasks used by the tactical groups were also presented and practiced demonstrating the need for a service rule, demonstrating how to win a point, using modified games to reinforce the long and short game, using left and right strategy, and hitting down on the shuttle to attack.

A few combination tasks that required students to practice a sequence of shots associated with a particular strategy (e.g., hit clear, then hit drop for long/ short game) were presented by the teacher. These combination tasks were presented using direct instruction. The teacher had planned to use a greater number of combination tasks. During student practice of combination tasks, students would begin on task and sometimes moved to hitting the shuttle back and forth cooperatively. The problem was not a management problem for the teacher. The students were largely well behaved and compliant with task instructions. The temptation to continue hitting the shuttle back and forth was very great for these beginning badminton players. Because there were a limited number of lessons available for instruction, the teacher was concerned that students were not maintaining focused practice on these combination tasks. The teacher did not use as many combination tasks as the teacher had originally planned.

During game play, the combination teacher provided feedback on both the tactics and skill technique used by students. On the last day of instruction in the combination group, students played an opponent and wrote on a piece of paper between points their plans for the next point, analysis of the opponent's strengths and weaknesses, and other tactics used during game play.

Treatment Verification. All three teachers audiotaped each of their lessons. Audiotapes were analyzed by trained observers. Analysis included the number and type of tasks for each lesson, the nature of teacher feedback, and the use of time. Observers counted the number of tasks per lesson and calculated an average based 
on the total number of lessons. Tasks were categorized by type or major focus into skill, strategy, and combination tasks. Percentages of each type of task for each teacher were based on the total number of tasks for each teacher.

A summary of the number of tasks per lesson, percentage of the types of tasks, feedback, and use of time is presented in Figure 1. The skill teacher averaged 3 tasks per lesson, with $100 \%$ of the tasks being oriented to skill instruction. The strategy teacher averaged 2.6 tasks per lesson, with $100 \%$ of the tasks being oriented to strategy instruction. The combination teacher averaged 2.0 tasks per lesson, with 33\% skill tasks, 58\% strategy tasks, and 9\% a combination of skill and strategy tasks.

Feedback for each teacher was counted by episode and categorized by positive, general, strategy, skill, behavioral, and task. Percentages for each teacher were calculated based on their total number of feedback episodes. Each teacher's use of time was analyzed and placed into categories of instruction, management, and activity or practice time. Simple percentages were calculated for each category for each teacher.

The feedback of each teacher reflected the particular treatment. The skill teacher had $41 \%$ of her feedback oriented to skill execution and another $12 \%$ devoted to the nature of the task. Only $8 \%$ of the skill teacher's feedback reflected game strategy. The strategy teacher focused $72 \%$ of feedback on game strategy, with only $6 \%$ relating to skill. The combination teacher focused equally on strat-
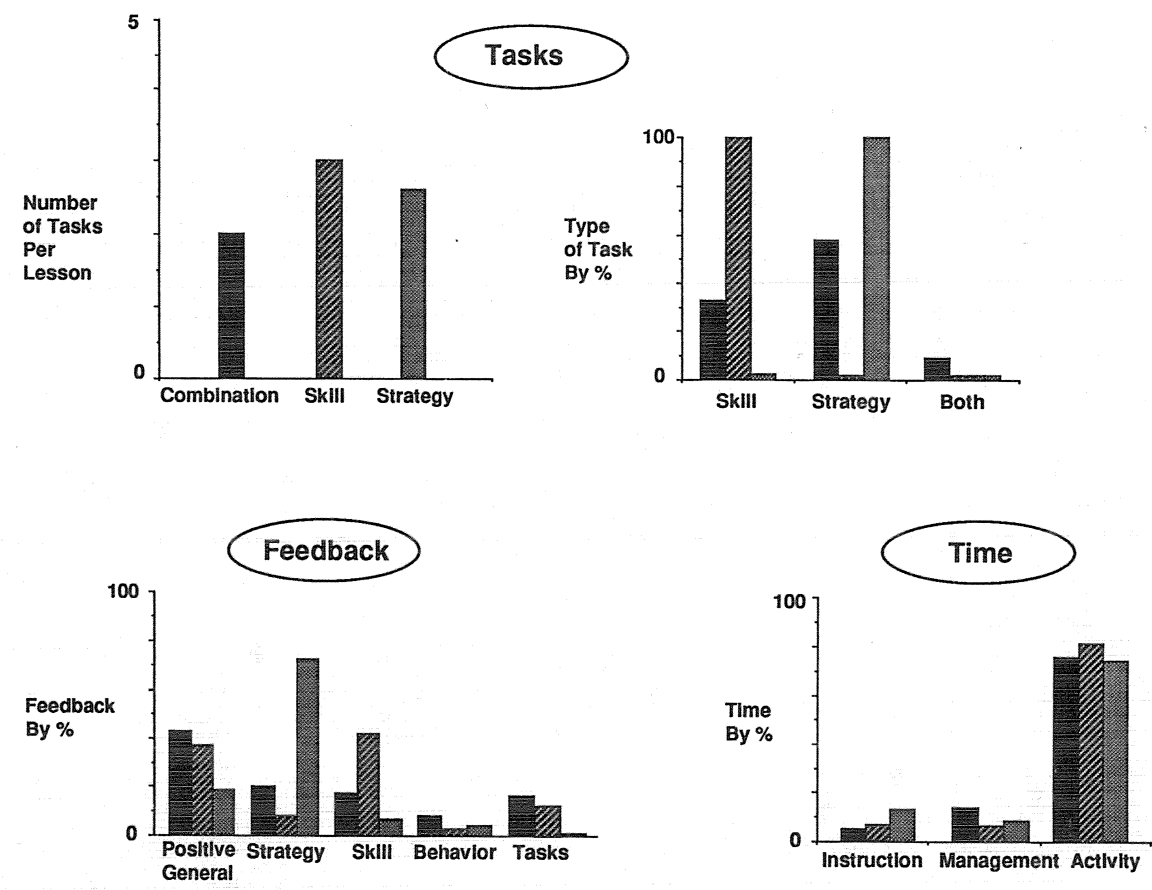

Figure 1 - Summary of teacher audiotapes. 
egy and skill feedback with $20 \%$ and $17 \%$, respectively. Moderate amounts of positive general feedback were given by all teachers: $37 \%, 19 \%$, and $42 \%$ for the skill, strategy, and combination teachers, respectively. Only a relatively small amount of behavioral feedback was given by each teacher as students were found to be cooperative and generally on task.

Time spent by each teacher emphasized efficient management, clear task presentations, and ample amount of student practice time. All teachers had $13.9 \%$ of time or less spent on management. Instruction time varied from $3 \%$ to $20 \%$ per lesson for each teacher, with an average near $10 \%$. Teachers' allotted practice time for students ranged from $65 \%$ to $93 \%$ per lesson, with an average near $80 \%$.

Teachers followed the intervention model they were assigned. Instruction was focused and goal oriented. Feedback was reflective of each intervention. Time was used to optimize learning.

\section{General Testing Procedures}

Badminton knowledge, skill, game play (videotaping), and the use of knowledge during performance were measured after a 2-day retention interval following the last day of instruction. All measures were collected over a 3-day period. Skill tests, game play, and point interviews were tested during the first 2 days of testing. All students took the written knowledge test in a classroom on the 3rd day of testing. More details of each test and procedures are provided in subsequent sections.

\section{Skill Tests Procedures}

Eight courts were positioned along the outside perimeter of the school gymnasium and were used for skill tests. Eight experimenters, two for each skill test (clear, drop, smash, serve), conducted all skill tests. Experimenters were trained in their respective test procedures in a 1.5-hour training session prior to data collection. Students were assigned at random to a prearranged order of skill tests and game play prior to testing. Thereafter, students rotated skill tests or game play stations during the testing. All students met as a group at the beginning of each testing session. General instructions for the day were explained. Students were individually called by an experimenter to the respective station (skill test or game play) where they would begin the testing. After a student had completed testing at that station, the experimenter directed the student to the next testing station in the rotation.

Each experimenter read a short set of written instructions to the student for each respective skill test. The student then proceeded to perform practice trials and the respective test.

Serve. The Poole Long-Serve Test (Johnson \& Nelson, 1986) was used to assess serve skill. The test required students to serve from the right service court to the right receiver's box. Scoring areas were marked in 16-inch zones starting from the 2 inches behind the baseline. Scores closest to the baseline were given the highest value. Point values for a trial ranged from 1 to 5 . Each student performed two practice trials followed by 10 test trials. The maximum score for an individual was 50 . More detailed procedures for the test are presented in Johnson and Nelson (1986).

Clear. The Poole Forehand Clear Test (Johnson \& Nelson, 1986) was used to assess the clear. The student stood at the center of the court, 2.5 feet from 
the baseline. The task required students to toss the shuttle and, using the forehand, hit the shuttle using the clear to four scoring areas on the other side of the net. Higher point values were associated with a deeper landing location of the shuttlecock on the court. Point values for an individual trial ranged from 1 to 4 . Two practice trials were performed followed by 10 test trials. The maximum score was 40. More detailed procedures for the test are presented in Johnson and Nelson (1986).

Smash. A test for smash skill was conducted similar to procedures used in the Badminton Smash Test (Johnson \& Nelson, 1986). The test reported in Johnson and Nelson required the use of a badminton set-up machine. The investigators constructed the machine using the specifications outlined by Johnson and Nelson. However, pilot tests with the machine were less than satisfactory. Thus, two experimenters were trained to reliably hit shuttles to the desired location with an appropriate trajectory to participants for this test. Each student stood in the center of the court. Experimenters hit the shuttlecock across the net and to the student. The student's task was to hit a smash (hit down) into one of two 4-foot, 4-inch wide target areas on each side of court. A trial that landed within one of these targets received a score of 2, whereas shuttles landing within court boundaries received a score of 1 . Shuttles landing out of bounds or in the net received a score of zero. Each student performed 2 practice trials followed by 10 test trials. The maximum score for an individual was 20 .

Drop. The two experimenters who administered the drop tests were trained to feed shuttles to students similar to procedures for the smash test. Each student stood near the center of the court. The student was instructed to return the shuttle using an overhand shot over the net into target areas. Three points were awarded for trials that landed just over the net into one of two 3 foot $\times 3$ foot target areas located in the front corners of the court. Two points were awarded for trials where the shuttle landed in front of the service line in the center of the court. One point was awarded for trials where the shuttle hit the top of the net but did not go over. Shuttles landing out of bounds or beyond the service line received a score of zero. The maximum score was 30 .

\section{Game Play Procedures}

All students, including those in the control group, were rated by their respective teacher to determine skill level. Control students were rated on general motor ability, whereas treatment students were rated on badminton skill. Students in the control and treatment groups were assigned to play an opponent of similar ability. Four regulation courts positioned in the center of the gym were used for game play. One experimenter was assigned to each court. Students were instructed to play badminton singles, were shown the court markings and boundaries, and were instructed to report their retrospective thoughts during play at the end of each point using a cassette recorder (see Point Interviews section). Students played a singles game for 10 minutes. Games were timed by one experimenter.

Each student's game play was videotaped by one of eight videocameras positioned high in the bleachers (four on each side). Each camera videotaped primarily one side of one court used for game play. Three experimenters positioned the cameras and monitored videotaping game play each day. 


\section{Game Play Instrument}

An observational instrument was designed to measure players' tactical and skill behavior during game play. Previous instruments (French \& Thomas, 1987, basketball; McPherson \& French, 1991; McPherson \& Thomas, 1989, tennis; French, Spurgeon, \& Nevett, 1995, baseball; Turner \& Martinek, 1995b, field hockey) developed to examine cognitive and skill components of game performance in other sports served as guides. In most sports, there is a sequential order of cognitive and sport skill events. For example, in badminton, a player must position him- or herself appropriately on the court, decide whether it is appropriate to hit the shuttle, decide what type of shot to use and where to place the shot, make clean contact with the shuttle, and successfully execute the shot. Categories of positioning, control of object, decision, and execution of skill have been used in the instruments previously cited.

Game play categories used during instrument development included the following: contact decision (i.e., decision to make contact), contact execution (i.e., control or contact with the shuttle), type of shot performed, player position on the court at racket contact, opponent position on the court at racket contact, return to home base, decision concerning where to hit the shuttle, and execution of the shot. Similar to the instrument developed for tennis (McPherson \& Thomas, 1989), serve decisions and execution were considered separate components from other aspects of game play. The three positioning categories (position of the player on the court at racket contact, opponent's position at racket contact, and return to home base) did not discriminate skillful performance because skillful and less skillful players exhibited different styles of play. Less skilled players were more likely to hit the shuttle back and forth to each other and rarely forced each other to move around the court. More skillful players forced their opponents to move more often. Both the skilled and unskilled players were often coded in the center of the court. Thus, the three categories for positioning were eliminated from the final instrument because they were not able to discriminate different styles in play. The final categories used for the instrument were contact decision, contact execution, type of shot, game decisions, game execution, serve decision, and serve execution.

Contact decision referred to the decision whether to attempt to make contact with the shuttle. This decision was coded as follows:

$1=$ Made contact with shuttle in court.

$1=$ Made no contact, shuttle out of bounds.

$1=$ Made no contact, shuttle served to wrong service court.

$0=$ Hit shuttle when it would have landed out of bounds.

$0=$ Hit shuttle when served to wrong service court.

$0=$ Did not hit shuttle when it lands in bounds.

$\mathrm{N}=$ Not appropriate, player is serving.

$\mathrm{T}=$ Did not have time to reach the shuttle, did not make contact with shuttle coded only to note the end of a point.

A contact execution refers to the ability to make clean contact with the shuttlecock, similar to control of the object reported in Turner and Martinek (1992) and McPherson and Thomas (1989). This was coded as follows: 
$1=$ Clean contact made, clear intent for a shot (shuttle may land in or out of bounds, or in net).

$0=$ Swing and miss.

$0=$ Mishit badly so that shuttle drops immediately (mishit that lands on same same side of court).

$0=$ Mishit that intent is unknown (i.e., looks like smash in unskilled, shuttle get carried in a downward motion, sometimes to the floor, sometimes in the net).

$\mathrm{N}=$ Not appropriate, player is serving.

The type of shot hit by a player during the game was coded as follows:

$1=$ Serve.

2 = Drop: must land in the front of the court, trajectory must be lower than 3 feet above the net (otherwise code 7 as bloop_very high trajectory).

3 = Clear: shot must land beyond half court, high trajectory (not flat).

4 = Smash: must be hit down forcefully and land in front half of court, usually preparatory movement precedes.

$5=$ Drive: a forceful shot hit with a flatter trajectory than clear, should have some force (otherwise code 7 bloop).

7 = Bloop shot: nonforceful shot, hit with forearm only motion, little backward arm and racket preparation, can be clear or drive trajectory (some skilled players use this form to hit a drop, if it lands in front of court, code drop if the trajectory is not real, real high).

Game decisions referred to the choice of shot selection and placement that forced an opponent to move on the court to make contact with the shuttle. These decisions were coded even when the shuttle did not cross the net or was out of bounds. These were coded as follows:

$2=$ Shot made opponent run to forehand, backhand, up.

$2=$ Shot that forced opponent to move 2 steps backward.

$2=$ Smash hit straight at person and jams him or her, too hard to return.

$2=$ Shot hit down (smash or drive) with force at opponent's feet that forced him or her to hit return upward (e.g., student smash, opponent defends with underhand clear).

$1=$ Made opponent move two or three steps to the forehand or backhand (step not a reach), some movement required.

1 = Made opponent move two or three steps up or forward to return (not a couple of steps up for a kill shot).

$1=$ Bloop shots with high trajectory, must make person move half the court left, right, or up.

1 = Made opponent move 1 step backward.

$0=$ Hit straight to opponent-nontactical.

$0=$ Set-up shot, high shot in center of court, opponent may shuffle forward to reach it, shot gives opponent opportunity for a kill shot.

$0=$ May take one or two shuffle steps to receive high shot (bloop), sometimes to hit forehand, or just to move when it wasn't necessary. 
Game execution referred to shots that landed in bounds or out of bounds during game play. These were coded as follows:

$1=$ Hit within boundaries of play and correct service area.

$0=$ Hit in net.

$0=$ Hit out of bounds.

$0=$ Hit to wrong service court.

Eight dependent measures were calculated from categories of the observational instrument. The total number of each shot (bloop, clear, smash, drop, drive, serve) was determined from the category type of shot. This classification was used in calculation of forceful shots and cooperative shots. Table 1 provides a summary of the definitions and formulas used to calculate each dependent variable.

Objectivity of the game play instrument was determined by coding a sample of 10 students' game play by two trained independent coders. The eight dependent variables were calculated based on the codes of each observer. Intraclass correlation coefficients were greater than .85 for each dependent variable. Game play for the remaining students was coded by one trained coder. Reliability of observation was determined by coding a sample of 10 students' play a second time at the end of all coding. Dependent variables were calculated for each time of coding and intraclass correlations were conducted for each dependent variable. Intraclass correlations were greater than .90 for each dependent variable.

\section{Knowledge Measures}

A 45-question knowledge test was developed from the McGee and Farrow (1987) badminton test battery. Questions were selected from the rules, technique, and strategy sections. All questions concerned singles play. Questions categorized as rules included history, terminology, etiquette, rules, and scoring. Questions concerning technique sampled the serve, serve reception, drop, and smash. Actions of different body parts, position of the racket follow-through, and correction of technique were also sampled. Strategy questions included general strategy, as well as offensive and defensive strategies. The knowledge test was administered to all students in a regular classroom by one experimenter.

\section{Knowledge Used During Game Performance}

During game play, each subject was asked to report his or her retrospective thoughts during the previous point. These interviews have been termed point interviews in other studies (McPherson, 1993a, 1993b, 1994; McPherson \& Thomas, 1989). Point interviews have been shown as an effective way to elicit use of knowledge during game play. Participants' statements can be treated as data and analyzed for the content, structure, and metacognitive processes used during play. The content (concepts accessed during game play) and processes (self-regulation of tactics and sport skills) provide insight into what knowledge attention is directed toward and accessed into working memory and how knowledge may be used during game play. Ericcson and Simon (1993) have shown that using retrospective accounts of participants' thoughts is a valid technique for making inferences about thought content and processes. More detailed information concerning 
Table 1 Summary of the Calculation for the Dependent Variables Calculated as Measures of Game Play

Measure Calculation

Forceful shots

Contact decisions

Contact executions

Game decisions

Game execution

Serve decisions

Serve execution

Cooperative shots
Total number of shots coded as clear, drive, or smash divided by the total number of shots played minus the total number of drop shots played (percentage of shots hit with force during game).

Total number of appropriate contact decisions (coded 1) divided by the total number of opportunities (percentage of good decisions to contact shuttle when appropriate).

Total number of clean contacts with the shuttle (coded 1) divided by the total number of opportunities (percentage of clean contact with the shuttle).

Total number of decisions (other than serving) that forced an opponent to move (coded 1 or 2) divided by the total number of opportunities (percentage of shots played that forced opponent movement).

Total number of shots that were hit over the net and in bounds (coded 1) divided by the total opportunities (percentage of shots that were hit in bounds).

Total number of serve decisions that forced the opponent to move to return the serve (coded 1 or 2 ) divided by the total serve opportunities (percentage of shots that forced opponent to move to return the serve).

Total number of serves that landed within the correct service court (coded 1) divided by the total number of serve opportunities (percentage of serves that landed or would have landed in correct court).

The total number of bloop shots (coded 7) hit directly to an opponent (decision coded 0) divided by the total number of shot opportunities (percentage of nonforceful shots hit directly to opponent). Important measure to distinguish styles of play noted during instrument development.

the theoretical basis, rationale, and appropriate procedures for conducting and interpreting these types of interviews is documented in Ericcson and Simon (1993) and McPherson's work (1993a, 1993b, 1994). A summary of the characteristic thought content and processes used by experts and novices during play is described in more detail in McPherson (1994).

Two studies also provide evidence that the interview procedures do not interfere with game performance (McPherson \& Thomas, 1989, tennis; Nevett, 1996, 
baseball). Observational measures of cognitive and skill components of game performance (McPherson \& Thomas, 1989) and coaches' ratings of player's decision making and skill execution during games (Nevett, 1996) were similar when subjects played with and without interviews during game play.

Interview Procedures. The same probes used by McPherson and Thomas (1989) and McPherson (1993a, 1993b, 1994) were used to elicit students' thoughts so that data could be compared with previous work. The two probes used in previous papers cited were the following: "What were you thinking about on that point?" and "What are you thinking about now?" The first probe requests a retrospective account of the student's thoughts during the previous point. The second probe requests the student to state current thoughts and possible planning for the next point.

Experimenters instructed each student to answer each question between each point during game play. Chairs were positioned behind the end line of each court. The two probe questions were written on a sheet of paper and taped to the back of the chair. A cassette recorder was placed in the chair and turned on throughout the testing. Students went back to the chair between each point and answered the two probes. Experimenters reminded students to state their thoughts after each point.

\section{Results}

\section{Skill and Knowledge Test Results}

A MANOVA was used to test for group differences on the smash, drop, clear, serve, and knowledge tests. The MANOVA was significant, Wilks's lambda $=.51$, eta square $=.49, F(12,95)=2.28, p<.05$. Univariate ANOVAs were used as follow-up tests. Group differences were found for the drop, $F(3,42)=4.83$, $p<$ .01 ; clear, $F(3,42)=2.92, p<.05$; and serve, $F(3,42)=4.76, p<.01$. The tests for the smash and knowledge were nonsignificant. The means, standard deviations, and results of Duncan's multiple range follow-up tests for these measures are presented in Table 2.

\section{Game Play}

Relations Among Game Play Measures. Correlations were calculated among game play measures and are presented in Table 3 . The percentage of forceful shots was strongly related to the percentage of appropriate game decisions. Thus, individuals who used more forceful shots (clear, smash, drive) also made their opponent move to receive shots or hit away from the opponent more often. Both the percentage of forceful shots and game decisions were highly negatively related to cooperative shots. Some students basically played cooperatively by hitting low force shots directly to their opponent. Other students were limited in their ability to produce forceful shots. Inability to produce forceful shots reduced the space of the court that an opponent had to defend (cannot hit a clear to back of court) or gave the opponent more time to reach the shuttle by hitting high trajectory low force shots (few drives or smashes). Thus, decision-making components of performance during game play in badminton were related to skill execution components of performance. 
Table 2 Means and Standard Deviations for Skill Tests

\begin{tabular}{lccccccccc}
\hline & \multicolumn{2}{c}{ Control } & \multicolumn{2}{c}{ Combination } & \multicolumn{2}{c}{ Strategy } & \multicolumn{2}{c}{ Skill } \\
Variable & $M$ & $S D$ & $M$ & $S D$ & $M$ & $S D$ & $M$ & $S D$ \\
& & & & & & & & & \\
& & & & & & & & & \\
Smash & 10.4 & 6.6 & 11.1 & 4.5 & 12.3 & 4.8 & 10.9 & 2.6 \\
Drop & $10.7^{\mathrm{a}}$ & 6.1 & $11.2^{\mathrm{a}}$ & 5.5 & $16.7^{\mathrm{b}}$ & 4.3 & $17.8^{\mathrm{b}}$ & 5.5 \\
Clear & $15.0^{\mathrm{a}}$ & 9.3 & $25.1^{\mathrm{b}}$ & 9.0 & $22.7^{\mathrm{b}}$ & 9.2 & $25.0^{\mathrm{b}}$ & 6.7 \\
Serve & $10.8^{\mathrm{a}}$ & 11.9 & $19.1^{\mathrm{a}, \mathrm{b}}$ & 10.1 & $24.2^{\mathrm{b}}$ & 10.7 & $25.2^{\mathrm{b}}$ & 5.2 \\
& & & & & & & & & \\
\hline
\end{tabular}

Note. Means with the same superscripts were identified as similar by Duncan's multiple range test.

Table 3 Correlations Between Measures of Game Play

\begin{tabular}{lccccccc}
\hline & $\begin{array}{c}\text { Forceful } \\
\text { shots }\end{array}$ & $\begin{array}{c}\text { Game } \\
\text { dec. }\end{array}$ & $\begin{array}{c}\text { Game } \\
\text { exec. }\end{array}$ & $\begin{array}{c}\text { Contact } \\
\text { dec. }\end{array}$ & $\begin{array}{c}\text { Contact } \\
\text { exec. }\end{array}$ & $\begin{array}{c}\text { Serve } \\
\text { dec. }\end{array}$ & $\begin{array}{c}\text { Serve } \\
\text { exec. }\end{array}$ \\
\hline Forceful shots & & & & & & \\
Game decisions & $.70^{*}$ & & & & & & \\
Game execution & $-.36^{*}$ & $-.37^{*}$ & & & & & \\
Contact decision & $.56^{*}$ & $.54^{*}$ & -.21 & & & & \\
Contact execution & $.51^{*}$ & $.46^{*}$ & -.03 & $.55^{*}$ & & \\
Serve decision & $.37^{*}$ & $.60^{*}$ & -.06 & .20 & .27 & & \\
Serve execution & $.55^{*}$ & $.59^{*}$ & -.28 & $.50^{*}$ & $.43^{*}$ & $.42^{*}$ & \\
Cooperative shots & $-.91^{*}$ & $-.88^{*}$ & $.41^{*}$ & $-.64^{*}$ & $-.56^{*}$ & $-.47^{*}$ & $-.60^{*}$ \\
\hline
\end{tabular}

${ }^{*} p<.05$.

Execution of shots (shots hit in bounds) was negatively related to forceful shots and game decisions. Individuals who used more forceful shots and attempted to make opponents move were more likely to hit the shuttle out of bounds or into the net. The percentage of cooperative shots was positively related to execution of shots, which indicates that students who played more cooperatively were more likely to keep the shuttle in play.

Game Play Analysis. MANOVA was used to determine group differences in game play measures. The percentage of cooperative shots was not included in the MANOVA due to very high correlations with other variables. A separate ANOVA was conducted for cooperative shots. The MANOVA was significant, Wilks's lambda $=.27$, eta square $=.73, F(21,92)=2.58, p<.01$. Univariate ANOVAs and Duncan's multiple range test were used as follow-up tests. Group differences were found on all measures. 
Table 4 Means, Standard Deviations, and $\boldsymbol{F}$ values for Measures of Game Play

\begin{tabular}{lrrrrrrrrr}
\hline & \multicolumn{1}{c}{ Control } & \multicolumn{1}{c}{ Combination } & \multicolumn{1}{c}{ Tactical } & \multicolumn{2}{c}{ Skill } \\
Variable & $M$ & $S D$ & $M$ & $S D$ & $M$ & $S D$ & $M$ & $S D$ & $F$ \\
& & & & & & & & & \\
\hline & & & & & & & & & \\
Forceful shots & $18.1^{\mathrm{a}}$ & 26.4 & $57.2^{\mathrm{b}}$ & 19.4 & $61.7^{\mathrm{b}}$ & 25.9 & $57.7^{\mathrm{b}}$ & 24.2 & $6.84^{* *}$ \\
Game decision & $19.6^{\mathrm{a}}$ & 17.9 & $47.7^{\mathrm{b}}$ & 16.2 & $57.0^{\mathrm{b}}$ & 12.4 & $51.5^{\mathrm{b}}$ & 12.3 & $12.59^{* *}$ \\
Game execution & $97.7^{\mathrm{a}}$ & 3.6 & $92.4^{\mathrm{a}, \mathrm{b}}$ & 6.4 & $87.5^{\mathrm{b}}$ & 7.6 & $92.7^{\mathrm{a}, \mathrm{b}}$ & 9.6 & $3.28^{*}$ \\
Contact decision & $79.6^{\mathrm{a}}$ & 17.4 & $91.9^{\mathrm{b}}$ & 6.3 & $90.4^{\mathrm{b}}$ & 6.4 & $93.4^{\mathrm{b}}$ & 9.3 & $3.46^{*}$ \\
Contact execution & $64.3^{\mathrm{a}}$ & 26.6 & $84.6^{\mathrm{b}}$ & 13.0 & $73.9^{\mathrm{a}, \mathrm{b}}$ & 8.2 & $81.5^{\mathrm{b}}$ & 14.3 & $2.92^{*}$ \\
Serve decision & $6.9^{\mathrm{a}}$ & 11.4 & $24.8^{\mathrm{b}}$ & 22.7 & $29.0^{\mathrm{b}}$ & 19.6 & $33.0^{\mathrm{b}}$ & 22.2 & $3.25^{*}$ \\
Serve execution & $23.3^{\mathrm{a}}$ & 31.1 & $73.5^{\mathrm{b}}$ & 10.5 & $78.7^{\mathrm{b}}$ & 20.8 & $71.7^{\mathrm{b}}$ & 18.8 & $14.25^{* *}$ \\
Cooperative shots & $75.9^{\mathrm{a}}$ & 28.5 & $33.7^{\mathrm{b}}$ & 21.4 & $22.3^{\mathrm{b}}$ & 14.5 & $26.7^{\mathrm{b}}$ & 19.6 & $12.66^{* *}$ \\
& & & & & & & & &
\end{tabular}

Note. Means with the same superscripts were identified as similar by Duncan's multiple range test.

${ }^{*} p<.05 .{ }^{* *} p<.01$.

The means, standard deviations, and $F$ values for each variable are presented in Table 4. The skill, tactical, and combination groups were similar to each other but significantly different from the control group on forceful shots, game decisions, contact decisions, serve decisions, serve execution, and cooperative shots. The tactical group had significantly lower game execution (shots hit in bounds) than the control group. However, game execution was similar among the three treatment groups, and all treatment groups had lower game execution percentages than the control group. The differences in game execution were partly due to the treatment groups' better decisions (attempting to make the opponent move) and more forceful shots than the control group. The tactical group, however, had greater difficulty keeping the shots in play.

Contact execution (racket contact) was significantly higher in the combination and skill groups than in the control group. The tactical group exhibited scores similar to both the control group and other treatment groups. These should be interpreted similar to the game execution findings. Attempting more forceful shots and decisions may lead to mistakes in racket contact. In addition, subjects in the treatment groups were playing an opponent also in the treatment groups who made better decisions and executed more forceful shots than the control group. Thus, some errors in racket contact for the treatment groups were due to attempting to return more difficult shots. However, the tactical group had lower scores than the other treatment groups.

\section{Data Reduction and Analysis of Interviews}

A sample of 6 students' point interviews from each group with equal representation of males and females were selected for analysis. Each student's responses were transcribed verbatim. Care was taken to note voice inflection that would characterize more emotional responses. To control the number of verbalizations for each student, 
the first 10 points for each student were analyzed for knowledge content. McPherson's (1993b, 1994) model of protocol analysis for sport knowledge was used as a framework. A concept was defined as a word or a phrase about response selection in game contexts. Concepts were identified and categorized as goal, condition, action, selfregulation, probability statements, do statements, and affective comments. The following provides a summary of definitions for each category of concepts:

- Goals: Units of information that specify the means by which a game is won. May specify the purpose of selecting an action or a condition.

1. Execution goals: Goals that refer to trying to execute a sport skill.

2. General strategy goals: Goals that refer to the individual player and or opponent. Only the general goal is stated, not the specific condition-action sequence for achieving the general strategy.

3. Win goals: Goals that refer to winning the game, winning a point, or beatbeating an opponent.

- Condition: Units of information that specify when or under what circumstances to apply an action or pattern of actions.

- Action: Units of information that refer to the action selected or pattern of actions selected. An action may be a motor response (clear) or a perceptual response (look).

- Specialized elaborations of actions.

1. Self-regulation: Unit of information that describes whether an individual carried out a given action.

2. Do statement: Unit of information that describes how to execute the action.

- Specialized metacognitive statements.

1. Prediction or probability comments: Units of information that predict future actions or patterns of actions within a game context.

- Affective or reactive comments: Units of information that specify emotional responses during game play.

Goal concepts are further defined in terms of their hierarchical nature as execution, general strategy, and winning. Note that definitions for goals, conditions, and actions are consistent with cognitive models (Anderson, 1982) of procedural knowledge as productions: goal, condition (if), and action (then). These definitions and categories were used to develop a coding system for all the participants' statements during game play. Table 5 presents the concepts and exemplars for concepts generated by all the students during game play. The exemplars reported are verbatim or paraphrased statements. Students' responses were represented in the following categories: (a) goals for execution, general strategy, and winning; (b) the conditions prior opponent's shot, opponent tendencies, opponent position, and game status; (c) the actions smash, clear, drop, serve, hit it, hit it with at least one feature, and player movement or position; and (d) self-regulation statements for skill execution and tactics selected during the point. Categories were also developed for information irrelevant to the game, not thinking during the game, and several affective reactions.

One experimenter trained in verbal protocol analysis read all transcripts and developed a code sheet for concept categories. This code sheet of concept categories and exemplars was used by two trained independent coders to categorize all statements made by a sample of 12 students. Percentage of agreement between coders 
Table 5 Summary of Concepts and Examples of Students' Statements During Point Interviews

Concepts

\section{Goals}

Execution goals

Goals related to player and opponent (general strategies)

Goals related to winning

\section{Conditions}

Opponent's prior shot

Opponent tendencies

Opponent position

Game status

\section{Actions}

Smash

Clear (hit it deep)

Drop (hit it short)

Serve

Hit it (no specific features)

Hit it plus at least one feature

Player movement or position

\section{Self-regulation}

Skill execution

Tactical

Irrelevant

Not thinking

\section{Affective reaction ${ }^{\mathrm{a}}$}

Processing game experience

Psyche up, motivate, effort

External attributions

Response to mistakes

Reaction to playing a female
Exemplars of concept

Hit shuttle; hit shuttle over; keep shuttle in play

Trying to move opponent back; move opponent up

Win the point; win the game; beat opponent

Opponent hit a clear; opponent hit it long; opponent smashed

Opponent hits it up to the net a lot; opponent seems to be playing in the back

Opponent was in the front; opponent was close to net Score of game; player who is serving

Hit it over opponent's head; hit it in the corner; hit it crosscourt

Playing back; move up; get back to the other side

It was a bad serve; hit it too far back; hit it out; I mishit it

Deep serve strategy worked

Thinking about a dance; thinking about girls

I try not to think much; pretty much not thinking

This is dumb; that point was easy; this is a long game; this is a silly game; this is boring; will I get another point; I don't want to do this anymore

Comeback has started; I've got to try on the slams

Birdies fly too high; the net is too high

Another whiff; try not to screw up again

I can't let a girl beat me; I am thinking she's a boy, I am a girl, I can beat her

${ }^{a}$ Voice in flection was important for these determinations. 
for classifying statements into concept categories was greater than $90 \%$ for each student coded. The remaining transcripts were coded by one trained experimenter.

Several measures have been reported in previous work to analyze the content and structure of concepts (McPherson 1993a, 1993b). Among the measures for content previously used were the total number and variety of goal, condition, and action statements. A measure of the percentage of total statements (relative frequency) made in given categories has also been used (McPherson, 1993a). Relative frequency of concepts accessed during game play allowed the researcher to determine which concepts (ideas, propositions) were brought to conscious awareness more frequently and accessed into working memory most often during game play. We chose to use relative frequency as the measure of knowledge content accessed during play because it provided the best description of the thoughts accessed most frequently and best represented the data.

The categories of concepts were collapsed into eight major categories that were reported most frequently by subjects: execution goals, general strategy goals, winning goals, all actions, all affective comments, self-regulation of actions, and conditions related to opponent's prior shot and game status. Only 1 or 2 students reported concepts for opponent tendencies, opponent position, irrelevant information, not thinking, and self-regulation of tactics. Means for relative frequency of concepts accessed most frequently during game play for each group in each major category are presented in Table 6 . No statistical analysis was performed. The means are presented descriptively. The control group tended to report a higher frequency of concepts related to execution goals (make contact, hit it) and affective statements than the treatment groups. Almost all of the affective statements made by students in the control group were related to processing of their experience of the game (see exemplars). Some students included both positive ("Easy game") and

Table 6 Relative Frequency of Concepts Accessed in Each Major Category for Each Group During Game Play

\begin{tabular}{lrrrr} 
Concept & Control & Combination & Skill & Tactics \\
\hline Goals & & & & \\
$\quad$ Execution & 22.2 & 4.7 & 0.0 & 3.0 \\
$\quad$ Player/opponent & 3.7 & 3.3 & 1.0 & 3.0 \\
$\quad$ Winning & 1.5 & 11.5 & 6.2 & 15.7 \\
Conditions & & & & \\
$\quad$ Prior shot & 10.7 & 16.1 & 9.1 & 7.5 \\
$\quad$ Game status & 7.5 & 10.8 & 18.8 & 2.8 \\
Actions & 17.3 & 31.5 & 30.2 & 52.8 \\
Self-regulation & & & & \\
$\quad$ Execution & 0.0 & 14.2 & 14.7 & 11.3 \\
Affect & 28.5 & 4.5 & 3.3 & 0.0 \\
& & & &
\end{tabular}


negative ("Game is not as easy as I thought") comments across points. The tactical group tended to report a higher frequency of action concepts than did the skill and combination group. All treatment groups reported higher frequencies of action concepts than did the control group.

The structure of knowledge for condition-action linkages or goal-action linkages is not reported because there were so few linkages reported by any student (three total). Most of the linkages were self-regulation of an action concept. The treatment groups were slightly more likely to pay attention to the success or failure of a given skill shot (self-regulation execution). However, the self-regulation statements reported were low-level statements with no labels or elaboration on why the error occurred.

Few students accessed general strategy goals. This concept would typically include many of the tactics actually taught in a games for understanding approach as described by Lawton and Werner (1992). For example, general strategy goals would include the following: hit away from opponent, make opponent move, make opponent hit up, and use deception. Very few of these were reported in any group. Even though considerable time was spent using this language during instruction for the tactical and combination groups, few students in any group accessed these concepts during game play.

\section{Discussion}

The discussion is organized into two major sections. The first section focuses on the results of the skill tests and game play results. The second section focuses on issues related to the results of the point interviews.

\section{Skill Tests and Game Play}

The skill, tactical, and combination groups exhibited better performance than the control group on decision-making components of performance (contact decisions, game decisions, and serve decisions) and some measures of skill execution (clear skill test, forceful shots, and serve execution) during game play. The treatment groups also played less cooperatively (more competitively) during game play than the control group.

Differences among the treatment groups emerged on skill-execution measures. The combination group had lower scores on the serve and drop skill tests than did the skill and tactical groups. The ability to make clean contact with the shuttle (contact execution) and keep the shuttle within court boundaries during game play were slightly lower (though not statistically different) in the tactical group than in the skill or combination groups.

The performance of the skill group on decision-making components of game performance and the tactical group's performance on skill-execution measures are important issues. Neither group received explicit instruction on these components. One reason the skill group may have exhibited decision performance similar to the tactical and combination groups was the high correlation between the ability to produce forceful shots and make shot selections (decisions) that forced opponents to move or play a defensive shot. Instruction that facilitated the ability to produce force (hit clear to the back of court, smash or drive to increase speed of shuttle) 
may have led to shots that were more difficult to defend. A clear would force an opponent to defend more court space, whereas the smash and drive would limit the time to move to a defensive position or respond defensively.

There is also evidence that some aspects of decision making during game play may be acquired without direct instruction just by playing the game. McPherson and French (1991) reported improvement in game decisions for a group of beginning tennis players who received primarily skill-related instruction with some opportunity to play the game. Some relatively simple level of decision-making performance may be acquired simply by the opportunity to play the game. However, Turner and Martinek (1995b) did not find improvement in decisions in modified field hockey games for a group that received primarily skill instruction with some opportunity to play the game. Thus, decision making may not improve just by playing the game in all sports.

Few studies emphasizing tactical instruction have found improvement in skill execution measures without some direct instruction of skill (Griffin et al., 1995; Mitchell, Griffin, \& Oslin, 1995). French and Thomas (1987) and Turner and Martinek (1992) did report some improvement in the ability to control the object. McPherson and French (1991) found some improvement in the volley, but not for other tennis skills. Only one study (Turner \& Martinek, 1995b) has found significant improvement in skill execution components of performance when the instruction was primarily tactically oriented. Explicit skill instruction was introduced and integrated with tactics when skill was limiting the tactics of students (Turner \& Martinek, 1995b).

Many of the tasks used in tactical instruction in this study (long and short game, hit shuttle down to attack) created a task environment which may have elicited practice of the type of movement pattern necessary to accomplish the task (clear, drop, smash). For example, one tactical task asked students to play a game where points could only be scored when the shuttle landed on the court behind the opponent. This task encouraged the ability to produce both a drop shot to move the opponent forward and a clear to hit the shuttle over the opponent's head. Thus, the tactical group did practice some tasks that would elicit the movement patterns that would achieve the task goal.

Dynamical systems theory (Kugler, Kelso, \& Turvey 1982) suggests that organization of movement patterns is influenced by the interaction of organismic, environmental, and task constraints. Several authors have suggested that task organizations influence movement patterns generated by individuals (Halverson, 1966; Herkowitz, 1978; Strohmeyer, Williams, \& Schaub-George, 1991). Tasks may also be designed to elicit more mature movement patterns. Some authors (Halverson, 1966; Rink, 1993; Siedentop, Herkowitz, \& Rink, 1984) have referred to this as environmental design. Several studies (Rink, French, Werner, Lynn, \& Mays, 1992; Sweeting \& Rink, in press) have shown that extension tasks or environmentally designed tasks do facilitate skill development. More research is needed to discover principles for how environmentally designed tasks may facilitate skill development.

The treatment groups were similar on most measures of skill and decision making during games. However, the combination group exhibited poorer performance on the drop and serve skill tests than did the tactical or skill groups. The combination group had to divide practice time and tasks between skill-related tasks and strategy-related tasks. The poorer performance on these tasks may be related 
to less time available for practice on these tasks. Thus, the effects of combined skill and tactical instruction need to be studied over a longer period of time.

\section{Point Interviews}

The point interview data provide insight into students' thoughts and affect during game play. The control group had more statements related to making contact with the shuttle (execution goals) and affect related to their feelings about playing the game. All treatment groups had fewer affective statements, fewer execution goals, more action concepts, and slightly more self-regulation of skill execution. The tactical group accessed more action concepts during the game than the skill or combination groups.

The actual content of knowledge accessed during game play for the treatment groups showed just how novice these players were after 3 weeks of instruction. Housner (1981) reported that expert badminton players often accessed condition-action linkages during game play. Students in this study reported almost no condition-action links, very few conditions other than literal ones (opponent's prior shot or game status), few features or elaborations for actions (isolated actions), a few self-regulation of skills with no elaboration or labels for explanation of the error, and very few general strategy goals. All of these characteristics are similar to the characteristics of novices reported by McPherson (1994). None of these students thought in sophisticated ways about their play.

One would have expected that students in the tactical and combination groups would have accessed general strategy goals more often than the skill or control groups because much of their instruction focused on awareness of general tactics (e.g., hit away from opponent, move opponent up, move opponent back, move opponent right or left, use deception). Few students in any group accessed these general tactics. Some students could demonstrate some of these tactics during game play. However, students did not access these tactics during point interviews. Thus, the decision performance exhibited in games was not accompanied by much improvement in the knowledge content or structure used during game play. Students in this study were just beginning to play competitively at the end of 3 weeks of instruction. Use of knowledge and metacognitive strategies during game play may take a longer period of time to develop or require different types of tactical instruction.

The only other instructional study (McPherson, 1991, 1992, 1994; McPherson $\&$ French, 1991) that used measures of game play and point interviews to compare tactical and skill approaches found similar trends for decision performance in game play in relation to knowledge used during game play. Decision performance during game play appeared to indicate adequate performance. The knowledge content, structure, and metacognitive processes used during game play were more characteristic of beginners and novices, even after an entire semester of instruction. The group that received tactical instruction had more advanced use of knowledge during play in comparison with the group that received skill instruction primarily (McPherson, 1991,1992). The ability to use knowledge and think in more sophisticated ways during play may take much longer to develop than simple decision performance during game play.

Tactical instruction in McPherson and French (1991; McPherson, 1991, 1992) focused more on developing condition-action (if-then) relationships using direct 
instruction. The tactical group in that study may have exhibited more advanced knowledge use during play than the skill group because the tactical instruction was made more explicit to the learner. In addition, instruction was over a longer period of time than the present badminton study. Differences between tactical and skill approaches to instruction in knowledge use during performance may only become apparent with longer periods of instruction.

Research in expertise (McPherson, 1994) is just beginning to describe and conceptualize the cognitive processes that performers use during game performance. An example of some of the processes experts use is illustrated by some of the concepts that individuals may access during performance. Only one participant in this study accessed conditions related to an opponent's tendencies (two concepts). Experts would likely access this concept much more often. The access of an opponent's tendency is an indication that the individual was monitoring the opponent's actions over the course of several points, encoding the opponent's offensive or defensive actions, retrieving those actions, and generalizing or predicting from those actions a set of tendencies for the opponent. The opponent's tendency may be used to plan future tactics and actions. This type of processing is characteristic of expert performers. Experts are more likely to monitor game conditions, use specialized encoding and retrieval strategies, formulate situational probabilities based on this information, and plan actions in advance to attack or defend (McPherson, 1993a, 1994; Thomas, French, \& Humphries, 1986).

Facilitating the acquisition of the processes noted above may require students to actively practice tasks that analyze opponents' weaknesses, strengths, and tendencies and that plan future tactics based on these analyses. Few tasks in this study were practiced by the tactical group and the combination group that specifically asked subjects to systematically analyze their opponent and plan future tactics (last day of instruction). These types of tasks were used more frequently in McPherson and French (1991). Some cooperative learning tasks where one player advised another player regarding tactics were also used in McPherson and French. More research is needed to understand how different instructional approaches and types of tasks may influence the development of more sophisticated use of knowledge content, structure, and metacognitive processes during game play.

\section{Characteristics of the Control Group}

The performance of the control group provides some insight into the problems encountered by beginners as they initially attempt to play badminton. Many of the problems of this group were related to making contact with the shuttle. Contact execution during game play was about $64 \%$. Essentially, they missed the shuttle or mishit it 4 out of every 10 attempts. A portion of this group's poor performance on the skill tests was also due to lack of ability to make contact with the shuttle. Not surprisingly, the control group focused more attention and thoughts during the point interviews on execution goals (make contact, hit it) and affective responses related to their feelings about the game. The control group played much more cooperatively. About $75 \%$ of their shot attempts were bloop shots right to an opponent. Riley and Roberton (1981) described the beginning stages of game play for children as cooperative. More research is needed to examine changes in game performance that may be associated with a developmental shift from cooperative play to more competitive play. 